\title{
H.264 VIDEO TRANSMISSION OVER WLAN IN OPNET MODELLER
}

\author{
Lukáš Sendrei — Ján Valiska — Stanislav Marchevský *
}

\begin{abstract}
One of the biggest challenges of communication networks is the video transmission in real time. It requires high demands on the available network capacity and transport mechanisms. Availability of smart mobile devices with batteries, which keep the terminal working for several hours, caused an increased interest in the research of the deployment of video transmission in wireless transmission systems. The presented paper deals with the transmission of video encoded with H.264/AVC (Advanced Video Coding) video coding standard through wireless local area network (WLAN) using the programming environment OPNET Modeller (OM). The test network studied in this work was prepared by combining real and simulated networks, which allows interesting possibilities when working with the OM tools. Such an approach to working with OM allows a detailed video streaming analysis, because the video output was noticeably not only in the form of statistics, but we can see the real impact of transmission failures. Using the OM simulation environment allows to design the transmission systems, which would be difficult to establish in laboratory conditions.
\end{abstract}

K e y w or d s: H.264/AVC, OPNET modeller, system-in-the-loop, video transmission, wireless local area network

\section{INTRODUCTION}

The development of wireless communication has been marked by a huge expansion in recent years. We deal with it every day. It simplifies our work, communication and entertainment. The achievements of modern mobile networks reach parameters which ensure quality delivery of much information to various applications. They found wide usage in many areas. This creates significant pressure on the efficient use of available resources according to the philosophy "transfer as much as possible for as little as possible".

Despite the advanced communication technology the research is also focused on the video coding compression algorithms. In this area, several standards for video coding were created. A number of research institutions, organisations, universities and also commercial companies participate in the time-consuming and difficult process of standardisation. One of the latest standards for video coding is H.264/AVC, which represents a significant movement in terms of reducing coding requirements and improves visual quality $[1,2]$.

In this paper we show the advantageous combination of a real network with a simulated WLAN network designed in OPNET Modeller environment. The real video transmission generated on video server was imported into the simulated network, transmitted through the simulated WLAN network and transported back to the real network, where the received video sequence was decoded. OPNET Modeller was implemented as a generator of statistical parameters.
This paper is organised as follows. The second part is dedicated to a brief review of the H.264/AVC video compression standard. The third part contains information about wireless network classification and a brief history of WLAN evolution. In the fourth part the OPNET Modeller is introduced and the possibilities of video transmission in this environment are described. Part five deals with the simulations and summarizes the results. The last section contains a brief summary.

\section{H.264/AVC}

H.264/AVC is an international video coding standard that was first published in 2003. The standard was created within the collaboration of the working group (WG) MPEG (Moving Picture Experts Group) of organization ISO/IEC and VCEG group (Video Coding Experts Group) of the ITU-T institute. This WG's created together a WG JVT (Joint Video Team) to finalize the project of ITU-T called H.264 and ISO/IEC project named MPEG-4 part 10, Advanced Video Coding. H.264/AVC represents the latest concept of video coding from the series of standards H.261, MPEG-1 Video, MPEG-2 Video, H.263 and MPEG-4 Visual/Part 2. The main aim of the standardisation effort was to achieve increased coding efficiency and provide a better adaptation to the transmission network for various applications like videoconference, video streaming, video on demand, TV broadcasting or video storage. Compared with older standards, H.264/AVC provides a more efficient compression of different bit rates and video resolution by $50 \%$ on av-

* Department of Electronics and Multimedia Communication, Faculty of Electrical Engineering and Informatics, Technical University of Košice, Letná 9, 04200 Košice, Slovakia, \{lukas.sendrei, jan.valiska, stanislav.marchevsky\}@tuke.sk 


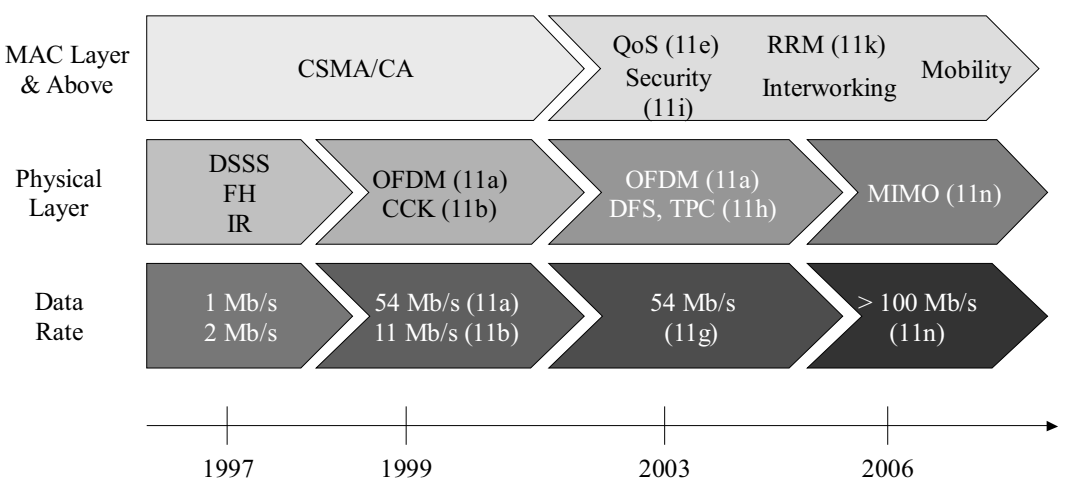

Fig. 1. Evolution of WLANs based on IEEE 802.11 technology

erage. But this resulted into a more complex decoder architecture $[1,3,4]$.

The standard defines the syntax and semantics of the bit stream as well as pre-processing which requires the decoder to correctly decode the bit stream to a viewable video. The standard does not define the H.264/AVC video coding or other pre-processing processes, enabling manufacturers to operate at a price, coding efficiency, error concealment, error resiliency or hardware requirements. Another area where different manufacturers can use their tools is posting of processing of the decoded video to create a video stream optimized for the target application [2]. The concept of H.264/AVC is based on two concept layers $[2,5]$ :

- VCL (Video Coding Layer) - executes the video coding itself,

- NAL (Network Abstraction Layer) - supports video transmission trough different types of networks.

The design of VCL is based on hybrid block video coding. The basic coding algorithm is a combination of inter frame prediction to exploit temporal statistical dependence and transform coding of pre-prediction residuals to exploit spatial statistical dependencies. In the VCL there is no single coding element present that can provide the most significant improvement in compression efficiency than in previous standards. It is mainly a number of small improvements that together produce significant efficiency gain of the video coding algorithm. NAL layer is designed to organize video VCL layer data and provides header information that is used for suitable video transmission over the transport layer or storage. It facilitates the ability of mapping the VCL data to transport layers of many transmission systems [1].

\section{WLAN}

Wireless networks allow people to communicate and access information and applications without cables. This introduces a degree of freedom in movement and ability to extend applications to various parts of buildings, cities, or nearly every place in the world. Wireless networks use radio waves or infrared light as a carrier of information and the communication medium is air. Most manufacturers have a wireless network interface and small aerial in almost every equipment, allowing easy use and mobility. Depending on the area the wireless networks are able to cover and the ability to satisfy various communicating entities we distinguish the following types of wireless networks [6]:

- Wireless Personal Area Network (WPAN),

- Wireless Local Area Network (WLAN),

- Wireless Metropolitan Area Network (WMAN),

- Wireless Wide Area Network (WWAN).

The WLAN industry has become one of the fastest growing segments of the communication industry. This development is due to the greater use of WLAN devices based on standards of the IEEE 802.11 series. WG IEEE 802.11 has developed several aspects of the dominant WLAN standards, including safety, Quality of Service (QoS) and cooperation with unlicensed technologies. The first standard called IEEE 802.11 was released into public after several years of research cycle. This standard specifies three physical technologies and a single MAC protocol to support 1 and $2 \mathrm{Mb} / \mathrm{s}$ transmission over a wireless medium. In September 1999 the organization published the IEEE standard 802.11b (IEEE 1999b) for WLAN in the $2.4 \mathrm{GHz}$ band, which supports data rate $11 \mathrm{Mb} / \mathrm{s}$. As for the modulation technique, Complementary Coded Keying (CKK) was selected based on Direct Sequence Spread Spectrum (DSSS). Also in September 1999 the specification 802.11a (IEEE 1999c) was ratified to support the WLAN in $5 \mathrm{GHz}$ band with a maximum transfer rate of $54 \mathrm{Mb} / \mathrm{s}$ by implementing technology Orthogonal Frequency Division Multiplex (OFDM). OFDM is also used in the following specification 802.11g (IEEE 2003a), which allows a maximum data transfer rate $54 \mathrm{Mb} / \mathrm{s}$ for WLAN in the $2.4 \mathrm{GHz}$ frequency band [7]. The latest standard $802.11 \mathrm{n}$ of this group uses Multiple Input Multiple Output (MIMO) technology and enables data transfer rates exceeding $100 \mathrm{Mb} / \mathrm{s}$. Figure 1 shows the development of WLAN technology. 


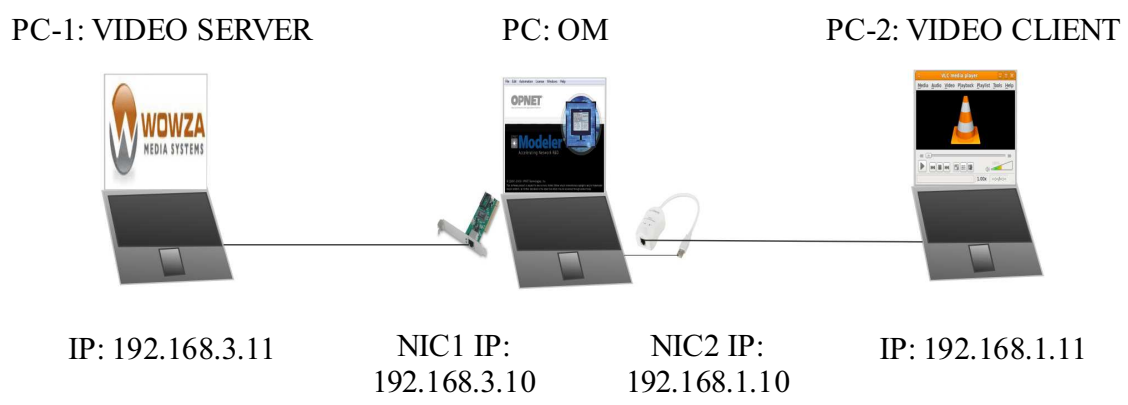

Fig. 2. Topology of the test network

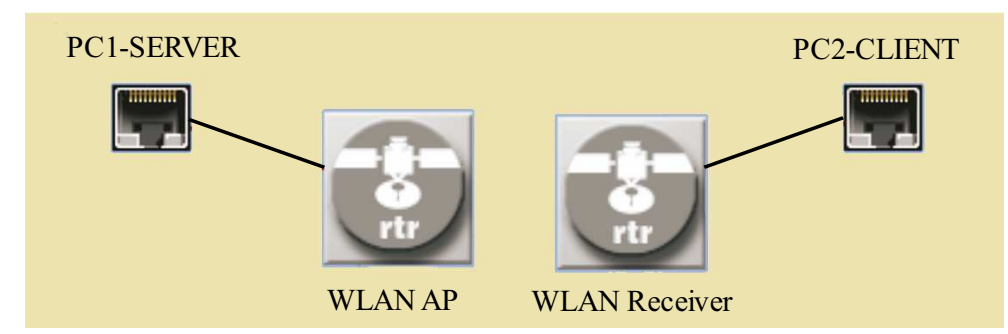

Fig. 3. Simulated WLAN in OM

\section{OPNET MODELLER}

OPNET Modeller is one of the most powerful simulation tools in the field of communication technologies. It is deployed in research and development of communication protocols and devices. Designers and administrators of communication networks can use this tool for efficiency analysis, optimisation, analysis of future growth and other properties of networks. Sophisticated network analysis suite of tools available for OPNET Modeller provides the opportunity to specify the models in details, identify significant individual components, perform simulations and analyse the generated output data [8].

System-in-the-loop (SITL) is a special module that allows connection of a simulated network in the OM environment and software applications or hardware of networks operating in real time. By using SITL during the simulation, packets are transmitted between the simulated and real network in real time. SITL Gateway in OM represents an external device which exchanges simulation packets and the WinPcap library in the operating system Windows is used for routing user-defined filter selected packets from the Ethernet network adapter in the simulation. The workstation on which OM runs, and hence the simulation, is connected to the real network via Ethernet interface. The simulation must run in real time to guarantee the synchronization and conversion of packet traffic flow inside the simulated system [9].

There are three basic network topologies for simulations with SITL:

- real-to-sim (communication between real and simulated network),
- real-sim-real (communication between real networks trough simulated network),

- sim-real-sim (communication between simulated networks through real network).

OM contains a range of predefined applications that can be adjusted as necessary. Video transmission is provided by the application named videoconferencing. We can set a frame size and frame rate and even some network parameters. This represents an opportunity to compile the required data flow model of video sequences, but does not allow visualization and change of the specific parameters of the video streams. An interesting solution in this respect is the connection of real systems with the simulation in the OM environment. Using this variant we can connect real network device that will generate and also receive real video streams to simulated network that will transport these streams during the simulation. According to the selected characteristics, type and topology the simulated network affects the transmission parameters of the real video content. Thus it is possible in the simulation environment to design very complex and extensive networks and it is easy to test them with real applications.

\section{SIMULATIONS}

Taking into account the facts mentioned above an approach that considers the connection between the real equipment and a simulated network in the OM environment has been chosen. SILT ensures connection between real workstations and the simulation. OM provided video 


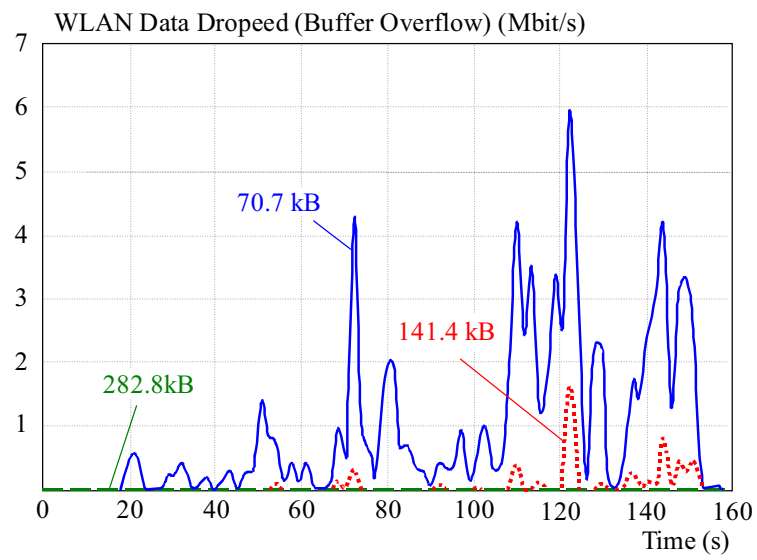

Fig. 4. WLAN buffer overflow (resolution $1920 \times 1080$ )

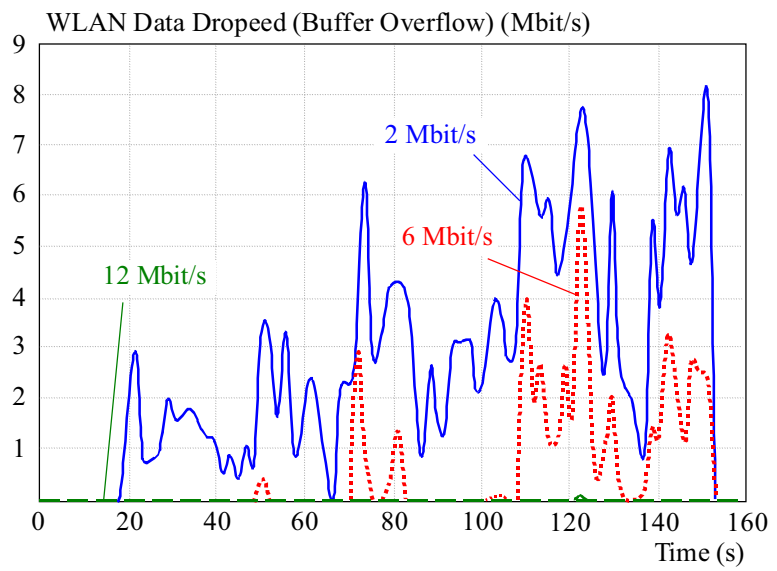

Fig. 6. Losses by low WLAN data rates (resolution $1920 \times 1080$ )

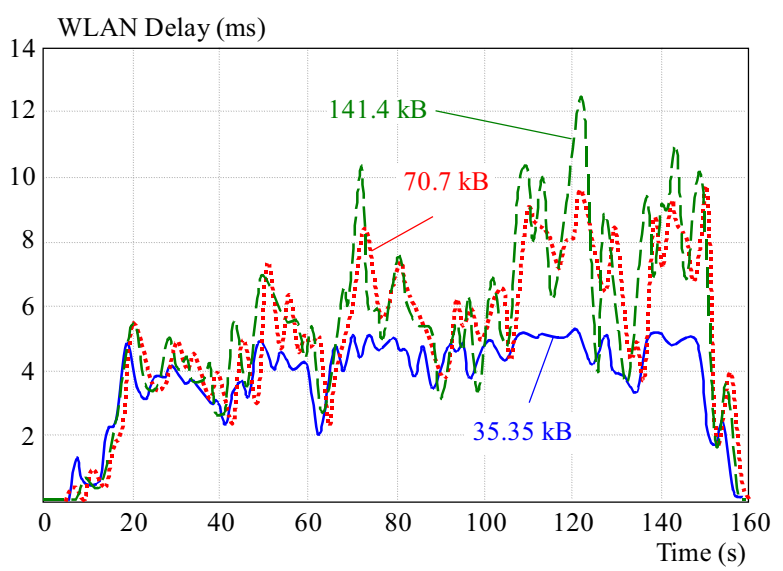

Fig. 8. WLAN delay (resolution $1280 \times 720$ )

transmission through the simulated WLAN. The real involvement topology consisted of three workstations. On the first workstation $P C-1: V I D E O S E R V E R$ there was the video server. As a video server software we deployed Wowza Media Server 3.1.0. It is a powerful streaming server which covers the delivery of video to various media players and devices. Workstation PC-2: VIDEO CLIENT displays and also stores the transmitted video using $V L C$ media player version 1.1.11. Workstation PC: OM, where

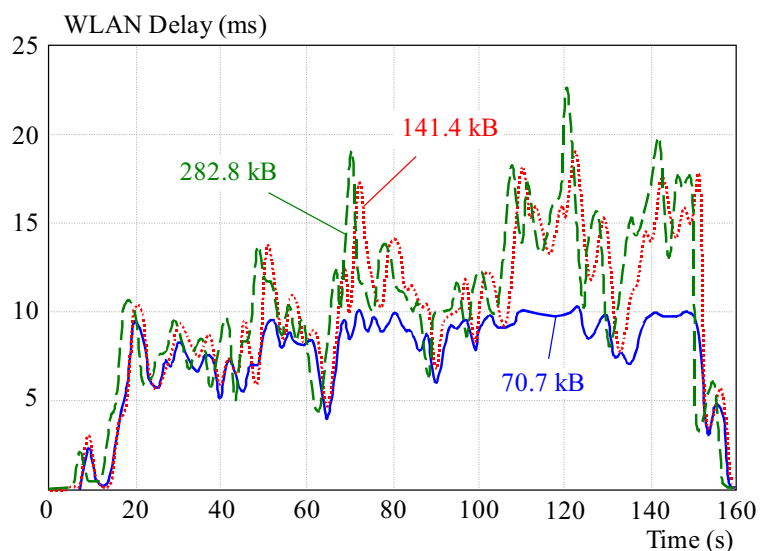

Fig. 5. WLAN delay (resolution $1920 \times 1080$ )

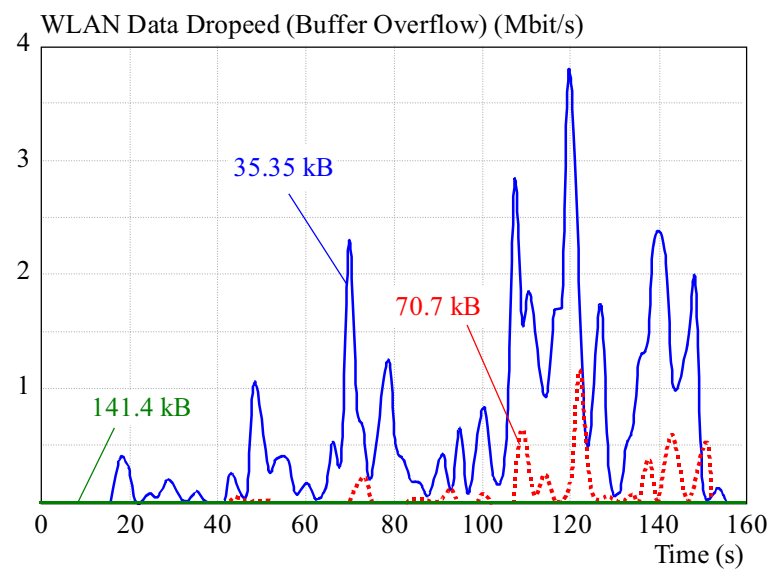

Fig. 7. WLAN buffer overflow (resolution $1280 \times 720$ )

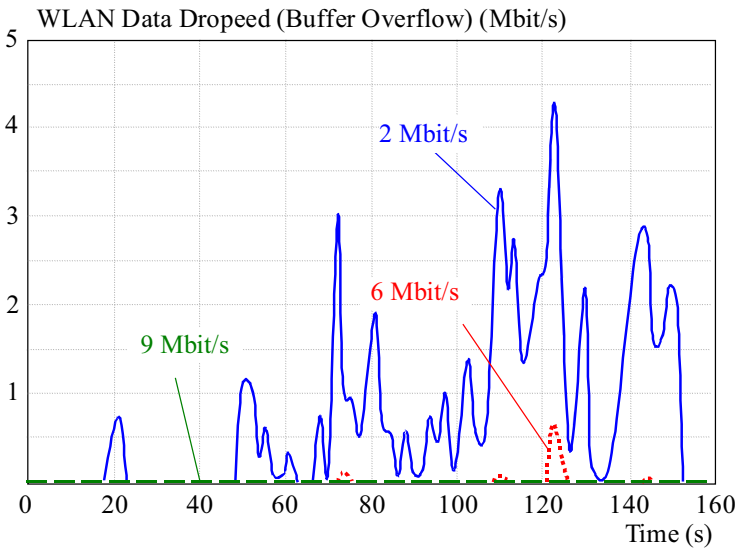

Fig. 9. Losses by low WLAN data rates (resolution $1280 \times 720$ )

OM was implemented, has two network interfaces, to which workstation server and client are connected. The topology of a test network is shown in Fig. 2.

The simulation was carried out in the programming environment OM. The simulated WLAN network was created in the OM project editor. The WLAN was composed of two wireless routers. The wireless router $W L A N A P$ was the WLAN Access Point (AP) and the AP functionality was disabled on the second WLAN 


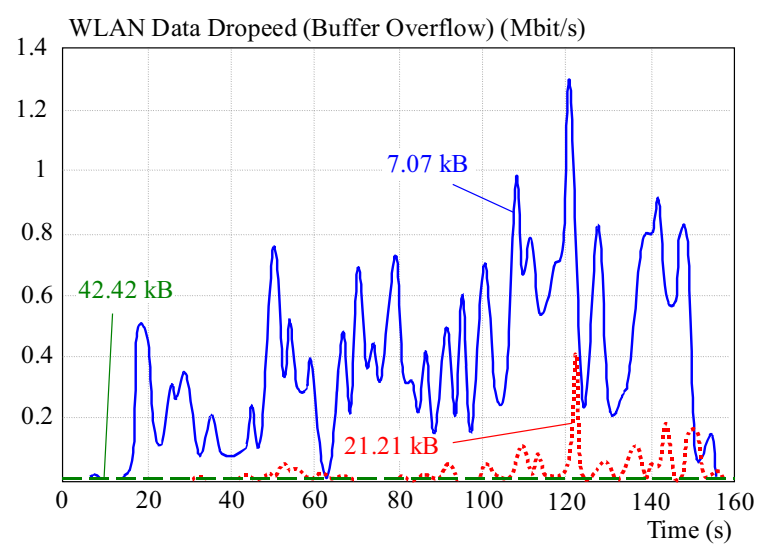

Fig. 10. WLAN buffer overflow (resolution $640 \times 360$ )

router WLAN Receiver. Both WLAN routers were connected using the special link sitl_virtual_eth_link with interface sitl_virtual_gateway_to_real_world. They are like sitl_virtual_eth_link part of the SITL module object library. Figure 3 shows the simulated WLAN network design in OM.

During the H.264/AVC video transmission simulations we used videos of different resolutions. Selected were three different resolutions, HD (High Definition) resolution $1920 \times 1280$ pixels, resolution $1280 \times 720$ and $640 \times 360$ pixels. The videos were converted using the $\mathrm{x} 264$ codec, which is freely available and is part of the popular media player $V L C$ player. All videos were saved in $m p 4$ format. In each simulation, the video was 3554 frames long and the frame rate was $24 \mathrm{fps}$ (frames per second). Video server created packets that are transported by UDP (User Datagram protocol). The maximum size of packets was $1414 \mathrm{~B}$. The video server sent video streams in the form of variable bit rate (VBR). VBR video stream can cause high losses in transmission, unless there is sufficient buffer size on network devices available, in this case on the wireless router. For video of various resolutions, the necessary buffer size varies considerably. By decreasing the resolution the demands on buffer size decrease as well. During the simulation we used WLAN IEEE 802.11g technology. WLAN transmission speed was $54 \mathrm{Mb} / \mathrm{s}$, with the exception of the video transmission testing at lower WLAN data rate. The main objective of the simulations was to determine the sufficient buffer size of the WLAN router and the minimum data WLAN data rate for error free video transmission over the simulated WLAN.

By resolution of $1920 \times 1080$ pixels we compared video transmission for three WLAN router buffer size: $70.7 \mathrm{kB}$, $141.4 \mathrm{kB}$ and $282.8 \mathrm{kB}$. The WLAN data dropped amount for three different buffer sizes for the transmission of HD video $(1920 \times 1080)$ is shown in Fig. 4. Sufficient memory size for lossless HD video transmission through WLAN at a resolution of $1920 \times 1080$ pixels is $282.8 \mathrm{kB}$. For this size and resolution we can achieve lossless transmission. The delay in the transmission of HD video $1920 \times 1080$ is visible in Fig. 5. The WLAN data dropped amount

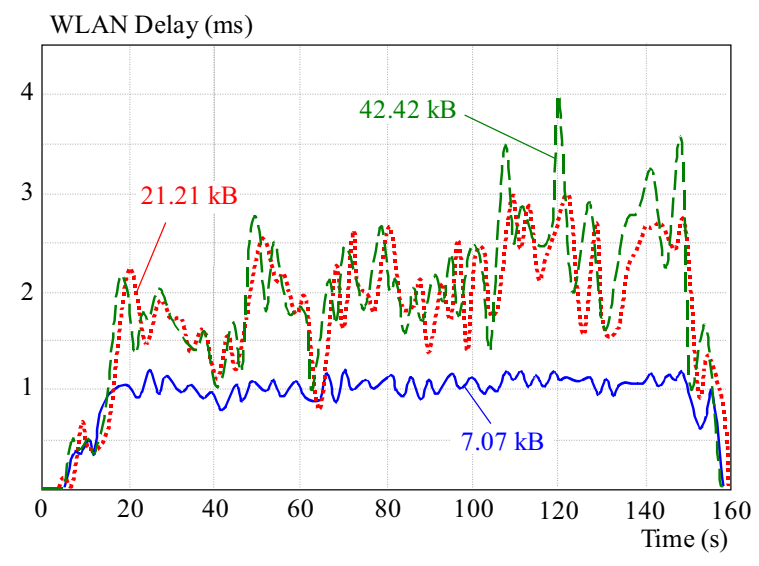

Fig. 11. WLAN delay (resolution $640 \times 360$ )

for HD video resolution $1920 \times 1080$ at low data rates is shown in Fig. 6. The results of the simulation show that the necessary transmission speed of wireless network is at least $12 \mathrm{Mb} / \mathrm{s}$.

Buffer size by the video transmission at lower resolution, namely $1280 \times 720$ pixels, was: $35.35 \mathrm{kB}, 70.7 \mathrm{kB}$ and $141.4 \mathrm{kB}$. Comparison of WLAN data dropped amount of the video stream is visible in Fig. 7 and the transmission delay in Fig. 8. This simulation shows that for lossless transmission of video of that resolution $(1280 \times 720)$ it is necessary to have a buffer size of $141.4 \mathrm{kB}$. Comparison of transmission losses at WLAN data rates 2, 6 and $9 \mathrm{Mb} / \mathrm{s}$ contains Fig. 9. The lowest bit rate, where significant losses were recorded was at $6 \mathrm{Mb} / \mathrm{s}$. The lowest bit rate, which causes no losses by video streaming through WLAN of this resolution, is $9 \mathrm{Mb} / \mathrm{s}$.

Properties of the transmission of low video resolution $640 \times 360$ pixels were tested for AP buffer size of: $7.07 \mathrm{kB}$, $21.21 \mathrm{kB}$ and $42.42 \mathrm{kB}$. Lossless transmission allows the size of $42.42 \mathrm{kB}$. Comparison of losses by the video transmission of resolution $640 \times 360$ pixels with varying buffer size is summarized in Fig. 10 and the relayed delay in Fig. 11. Resolution $640 \times 360$ has low bandwidth requirements as reflected in the results of subsequent simulations. Relatively large losses and the associated delays are still reported at a rate of $1 \mathrm{Mb} / \mathrm{s}$. Almost lossless transmission is possible by transmission speed of $2 \mathrm{Mb} / \mathrm{s}$ and completely lossless transmission at $5.5 \mathrm{Mb} / \mathrm{s}$. Results of the loss comparison at WLAN data rate 1,2 and $5.5 \mathrm{Mb} / \mathrm{s}$ for low resolution $640 \times 360$ pixels is shown in Fig. 12 .

\section{CONCLUSION}

The amount of simulated WLAN load scenarios with different parameter settings showed the possibilities and limitations in the video transmission of various resolutions in the OM environment. H.264/AVC standard has proven to be a suitable video encoding format to ensure the highest possible video streams transmission quality with relatively low requirements on the communication network. An important factor has been presented according to which there have to be sufficient network resources 
WLAN Data Dropeed (Buffer Overflow) (Mbit/s)

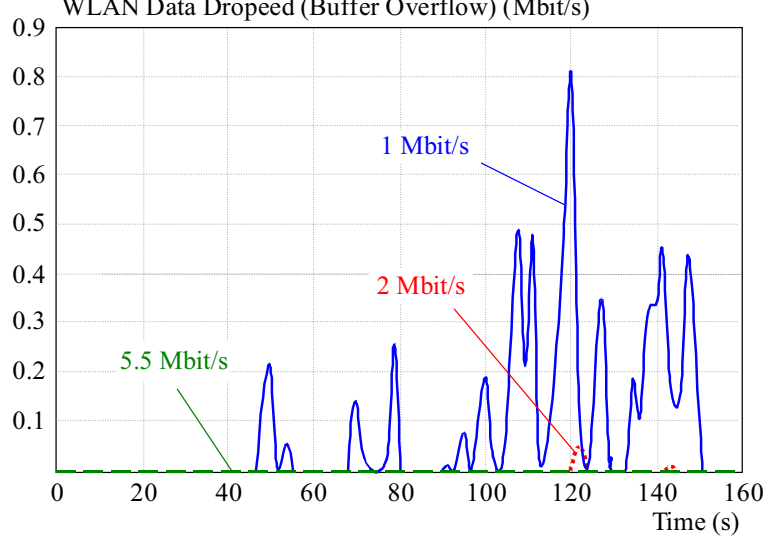

Fig. 12. Losses by low WLAN data rates (resolution $640 \times 360$ )

in terms of WLAN data rate and adequate buffer memory size for handling the VBR video. Shortcomings of the transmission of VBR video stream could be removed either in the process of video encoding, or by ensuring fixed bit rate on the video server site. That solution would reduce the load of network components. The delay variations can be sufficient and in balance using the cache on the video decoder. The only problem at the current state of technology which cannot be satisfactorily resolved is the transfer of HD video at low bit rates.

\section{REFERENCES}

[1] WIEGAND, T.-SUlLivan, G. J.-BJONTEGAARD, G.LUTHRA, A. : Overview of the H.264/AVC Video Coding Standard, IEEE Transactions on Circuits and Systems for Video Technology 13 No. 7 (July 2003), 560-576.

[2] OSTERMANN, J.-BORMANS, J.-LIST, P.-MARPE, D.NARROSCHKE, M.-PEREIRA, F.-STOCKHAMMER, T.WEDI, T. : Video Coding with H.264/AVC: Tools, Performance, and Complexity, IEEE Circuits and Systems Magazine 4 No. 1 (Apr 2004), 7-28.

[3] RICHARDSON, I. E. : H.264 and MPEG-4 Video Compression, Video Coding for Next-Generation Multimediapubl John Wiley \& Sons Ltd, The Atrium, Southern Gate, Chichester, West Sussex, 2003

[4] WIEGAND, T.-SCHWARZ, H.-JOCH, A.-KOSSENTINI, F. : Rate-Constrained Coder Control and Comparison of Video
Coding Standards, IEEE Transactions on Circuits and Systems for Video Technology 13 (July 2003), 688-703.

[5] GHANDI, M.-GHANBARI, M. : The H.264/AVC Video Coding Standard for the Next Generation Multimedia Communicationsjour J. Iranian Association Elec. Eng.

[6] GEIER, J. : Wireless Networks: First Step, Cisco, Indianapolis, 2004.

[7] ETOH, M.: Next Generation Mobile Systems 3G and Beyond, John Wiley \& Sons Ltd, The Atrium, Southern Gate, Chichester, West Sussex PO19 8SQ, England, 2005.

[8] MOHORKO, J.-MATJAZ, F.-SASA, K.: Advanced Modelling and Simulation Methods for Communication Networks, Microwave Review (Sep 2008).

[9] OPNET Technologies Inc. OPNET Modeller Product Documentation Release 14.5, OPNET Modeller, 2008.

Received 1 September 2012

Lukáš Sendrei born in 1987, graduated (MSc) with honours at the Department of Electronic and Multimedia Communication of the Faculty of Electrical Engineering and Informatics at Technical University in Košice, in 2012. He is a $\mathrm{PhD}$ student at the Department of Electronics and Multimedia Communications at the same university since September 2012. His current research interests include wireless communication and multicarrier communications systems.

Ján Valiska born in 1986, in Michalovce, received MSc degree at the Technical University of Košice. He graduated from Department of Electronics and Multimedia Communications, Faculty of Electrical Engineering and Informatics in May 2011. He is a PhD student at the Department of Electronics and Multimedia Communications at the same university since September 2011. His current research interests include image processing, object tracking, digital television systems and satellite communications.

Stanislav Marchevský received MSc in electrical engineering at the Faculty of Electrical Engineering, Czech Technical University in Prague, in 1976 and PhD degree in radioelectronics at the Technical University of Košice in 1985. Currently he is a Professor of Electronics and Multimedia Communications Department of Faculty of Electrical Engineering and Informatics of Technical University of Košce. He teaching interests include switching theory, digital television technology, and satellite communications. His research interests include image nonlinear filtering, neural networks, genetic algorithms, and multiuser detection, space-time communication, diversity communication over fading channel, and power and bandwidth efficient multiuser communications. 